Commun. Korean Math. Soc. 28 (2013), No. 1, pp. 135-141

http://dx.doi.org/10.4134/CKMS.2013.28.1.135

\title{
CONVERGENCE OF ISHIKAWA'METHOD FOR GENERALIZED HYBRID MAPPINGS
}

\author{
FAngfang Yan, Yongfu Su, And Qinsheng Feng
}

\begin{abstract}
In this paper, we first talk about a more wide class of nonlinear mappings, Then, we deal with weak convergence theorems for generalized hybrid mappings in a Hilbert space.
\end{abstract}

\section{Introduction}

Let $C$ be a nonempty closed convex subset of a real Hilbert space $H$. Then a mapping $T: C \rightarrow C$ is said to be nonexpansive if $\|T x-T y\| \leq\|x-y\|$ for all $x, y \in C$. The set of fixed points of $T$ is denoted by $F(T)$. A mapping $T: C \rightarrow C$ with $F(T) \neq \emptyset$ is called quasi-nonexpansive if $\|x-T y\| \leq\|x-y\|$ for all $x \in F(T)$ and $y \in C$. It is well-known that the set $F(T)$ of fixed points of a quasi-nonexpansive mapping $T$ is closed and convex; see [10].

A important example of nonexpansive mappings in a Hilbert space is a firmly nonexpansive mapping if

$$
\|F x-F y\|^{2} \leq\langle x-y, F x-F y\rangle
$$

for all $x, y \in C$; see for instance, $[3,5]$. It is known that a mapping $F: C \rightarrow C$ is firmly nonexpansive if and only if

$$
\|F x-F y\|^{2}+\|(I-F) x-(I-F) y\|^{2} \leq\|x-y\|^{2}
$$

for all $x, y \in C$, where $I$ is the identity mapping on $H$. It is also known that a firmly nonexpansive mapping $F$ can be deduced from an equilibrium problem in a Hilbert space; see, for instance, $[2,4]$.

Recently, Kohsaka and Takahashi [11] introduced the following nonlinear mapping: Let $E$ be a smooth, strictly convex and reflexive Banach space, let $J$ be the duality mapping of $E$ and let $C$ be a nonempty closed convex subset of $E$. Then, a mapping $S: C \rightarrow C$ is said to be nonspreading if

$$
\phi(S x, S y)+\phi(S y, S x) \leq \phi(S x, y)+\phi(S y, x)
$$

Received March 20, 2012.

2010 Mathematics Subject Classification. 47H10.

Key words and phrases. generalized hybrid mappings, Ishikawa's iteration, weak convergence, Hilbert space. 
for all $x, y \in C$, where $\phi(x, y)=\|x\|^{2}-2\langle x, J y\rangle+\|y\|^{2}$ for all $x, y \in E$. They consider such a mapping to study the resolvents of a maximal monotone operator in the Banach space. In the case when $E$ is a Hilbert space, we know that $\phi(x, y)=\|x-y\|^{2}$ for all $x, y \in E$. So, a nonspreading mapping [11] $S: C \rightarrow C$ in a Hilbert space $H$ is defined as follows:

$$
2\|S x-S y\|^{2} \leq\|S x-y\|^{2}+\|x-S y\|^{2}
$$

for all $x, y \in C$. A mapping $T: C \rightarrow C$ is called hybrid mapping [18] if

$$
3\|T x-T y\|^{2} \leq\|x-y\|^{2}+\|T x-y\|^{2}+\|T y-x\|^{2}
$$

for all $x, y \in C$. They proved fixed point theorems for such mappings; see also [12] and [8]. Aoyama et al. [1] introduced a class of nonlinear mappings called $\lambda$-hybrid and obtained a generalization of Baillons nonlinear ergodic theorem; see also [19]. Kocourek et al. [13] introduced a more wide class of nonlinear mappings containing the class of hybrid mappings. They called such mappings generalized hybrid mappings. Let $C$ be a nonempty, closed and convex subset of $H$. A mapping $T: C \rightarrow C$ is called generalized hybrid [22] if there exist $\alpha, \beta \in \mathbb{R}$ such that

$$
\alpha\|T x-T y\|^{2}+(1-\alpha)\|x-T y\|^{2} \leq \beta\|T x-y\|^{2}+(1-\beta)\|x-y\|^{2}
$$

for all $x, y \in C$. We call such a mapping an $(\alpha, \beta)$-generalized hybrid mapping. It is well known that the $(\alpha, \beta)$-generalized hybrid mapping is quasinonexpansive if the set of fixed points of it is nonempty. They proved fixed point theorems for such mappings; see [13]. We also observe that the mappings above generalize several well-known mappings. For example, an $(\alpha, \beta)$ generalized hybrid mapping is nonexpansive for $\alpha=1$ and $\beta=0$, nonspreading for $\alpha=2$ and $\beta=1$, and hybrid for $\alpha=\frac{3}{2}$ and $\beta=\frac{1}{2}$.

There classical iteration processes are often used to approximate a fixed point of a nonexpansive mapping. The first one is introduced by Halpern [6] and is defined as follows: Take an initial guess $x_{0} \in C$ arbitrarily and defined $\left\{x_{n}\right\}$ recursively by

$$
x_{n+1}=t_{n} x_{0}+\left(1-t_{n}\right) T x_{n}, \quad n \in \mathbb{N} \cup\{0\},
$$

where $\left\{t_{n}\right\}_{n=1}^{\infty}$ is a sequence in the interval [0,1]. The second iteration process is now known as Mann's iteration process [14] which is defined as

$$
x_{n+1}=\alpha_{n} x_{n}+\left(1-\alpha_{n}\right) T x_{n}, \quad n \in \mathbb{N} \cup\{0\},
$$

where the initial guess $x_{0}$ is taken in $C$ arbitrarily and the sequence $\left\{\alpha_{n}\right\}_{n=1}^{\infty}$ is in the interval $[0,1]$. The third iteration process is referred to as Ishikawa's iteration process [9] which is defined recursively by

$$
\left\{\begin{array}{l}
y_{n}=\beta_{n} x_{n}+\left(1-\beta_{n}\right) T x_{n}, \\
x_{n+1}=\alpha_{n} x_{n}+\left(1-\alpha_{n}\right) T y_{n},
\end{array}\right.
$$

where the initial guess $x_{0}$ is taken in $C$ arbitrarily, $\left\{\alpha_{n}\right\}_{n=1}^{\infty}$ and $\left\{\beta_{n}\right\}_{n=1}^{\infty}$ are sequences in the interval $[0,1]$. 
In this paper, we also use these iteration processes or it's evolvement to finish our proof.

\section{Preliminaries}

Throughout this paper, we denote by $\mathbb{N}$ the set of positive integers and by $\mathbb{R}$ the set of real numbers. Let $H$ be a real Hilbert space with inner produce $\langle\cdot, \cdot\rangle$ and norm $\|\cdot\|$. Let $C$ be a nonempty closed convex subset of a Hilbert space $H$. The nearest point projection of $H$ onto $C$ is denoted by $P_{C}$, that is, $\left\|x-P_{C} x\right\| \leq\|x-y\|$ for all $x \in H$ and $y \in C$. Such $P_{C}$ is called the metric projection of $H$ onto $C$. We know that the metric projection $P_{C}$ is firmly nonexpansive, i.e.,

$$
\left\|P_{C} x-P_{C} y\right\|^{2} \leq\left\langle P_{C} x-P_{C} y, x-y\right\rangle
$$

for all $x, y \in H$. Further $\left\langle P_{C} x-P_{C} y, x-y\right\rangle \leq 0$ holds for all $x \in H$ and $y \in C$; see, for instance, [16]. A Hilbert space satisfies Opials condition [15], that is,

$$
\liminf _{n \rightarrow \infty}\left\|x_{n}-u\right\|<\liminf _{n \rightarrow \infty}\left\|x_{n}-v\right\|
$$

if $x_{n} \rightarrow u$ and $u \neq v$; see [15].

We give the crucial lemmas in order to prove the main theorem.

Lemma 2.1 ([7]). Let $H$ be a real Hilbert space. Then for all $x, y \in H$ and $\alpha \in[0,1]$ the following inequality hold:

$$
\|\alpha x+(1-\alpha) y\|^{2}=\alpha\|x\|^{2}+(1-\alpha)\|y\|^{2}-\alpha(1-\alpha)\|x-y\|^{2} .
$$

Lemma 2.2 ([18]). Let $H$ be a Hilbert space and let $S$ be a nonempty closed convex subset of $H$. Let $\left\{x_{n}\right\}$ be a sequence in $H$. If $\left\|x_{n+1}-x\right\| \leq\left\|x_{n}-x\right\|$ for all $n \in \mathbb{N}$ and $x \in S$, then $\left\{P_{S}\left(x_{n}\right)\right\}$ converges strongly to some $z \in S$, where $P_{S}$ stands for the metric projection on $H$ onto $S$.

Using Opials theorem [15], we can also prove the following lemma; see, for instance, [17].

Lemma 2.3. Let $H$ be a Hilbert space and let $\left\{x_{n}\right\}$ be a sequence in $H$ such that there exists a nonempty subset $S \subset H$ satisfying (i) and (ii):

(i) For every $x^{*} \in S, \lim _{n \rightarrow \infty}\left\|x_{n}-x^{*}\right\|$ exists:

(ii) If a subsequence $\left\{x_{n_{j}}\right\} \subset\left\{x_{n}\right\}$ converges weakly to $x^{*}$, then $x^{*} \in S$.

Then there exists $x_{0} \in S$ such that $x_{n} \rightarrow x_{0}$.

\section{Weakly convergence theorem}

We are now in a position to prove our main theorem for weakly convergence of generalized hybrid mappings in a Hilbert space. 
Theorem 3.1. Let $C$ be a nonempty, closed convex subset of a real Hlibert space $H$. Let $T: C \rightarrow C$ be a generalized hybrid mapping with $F(T) \neq \emptyset$. Suppose that $\left\{x_{n}\right\}$ is a sequence generated by $x_{0}=x \in C, u \in C$ and

$$
\left\{\begin{array}{l}
y_{n}=\left(1-\beta_{n}\right) x_{n}+\beta_{n} T x_{n}, \\
x_{n+1}=\left(1-\alpha_{n}\right) x_{n}+\alpha_{n} T y_{n}
\end{array}\right.
$$

where $\left\{\alpha_{n}\right\}_{n=1}^{\infty}$ and $\left\{\beta_{n}\right\}_{n=1}^{\infty}$ are sequences of $[0,1]$ with $\liminf _{n \rightarrow \infty} \beta_{n}(1-$ $\left.\beta_{n}\right)>0$ and $0<a<\alpha_{n}<b<1$. Then $\left\{x_{n}\right\}$ converges weakly to $x_{0} \in F(T)$, where $x_{0}=\lim _{n \rightarrow \infty} P_{F(T)}\left(x_{n}\right)$.

Proof. Since $F(T) \neq \emptyset, T$ is quasi-nonexpansive. So, we have that for all $q \in F(T)$ and $n \in \mathbb{N}$ We have

$$
\begin{aligned}
& \left\|y_{n}-q\right\|^{2} \\
= & \left(1-\beta_{n}\right)\left\|x_{n}-q\right\|^{2}+\beta_{n}\left\|T x_{n}-q\right\|^{2}-\beta_{n}\left(1-\beta_{n}\right)\left\|x_{n}-T x_{n}\right\|^{2} \\
\leq & \left(1-\beta_{n}\right)\left\|x_{n}-q\right\|^{2}+\beta_{n}\left\|x_{n}-q\right\|^{2}-\beta_{n}\left(1-\beta_{n}\right)\left\|x_{n}-T x_{n}\right\|^{2} \\
= & \left\|x_{n}-q\right\|^{2}-\beta_{n}\left(1-\beta_{n}\right)\left\|x_{n}-T x_{n}\right\|^{2}
\end{aligned}
$$

and hence

$$
\begin{aligned}
& \left\|x_{n+1}-q\right\| \\
= & \left\|\left(1-\alpha_{n}\right) x_{n}+\alpha_{n} T y_{n}-q\right\| \\
= & \left(1-\alpha_{n}\right)\left\|x_{n}-q\right\|^{2}+\alpha_{n}\left\|T y_{n}-q\right\|^{2}-\alpha_{n}\left(1-\alpha_{n}\right)\left\|x_{n}-T y_{n}\right\|^{2} \\
\leq & \left(1-\alpha_{n}\right)\left\|x_{n}-q\right\|^{2}+\alpha_{n}\left\|y_{n}-q\right\|^{2}-\alpha_{n}\left(1-\alpha_{n}\right)\left\|x_{n}-T y_{n}\right\|^{2} \\
\leq & \left(1-\alpha_{n}\right)\left\|x_{n}-q\right\|^{2}+\alpha_{n}\left\|x_{n}-q\right\|^{2}-\alpha_{n} \beta_{n}\left(1-\beta_{n}\right)\left\|x_{n}-T x_{n}\right\|^{2} \\
& -\alpha_{n}\left(1-\alpha_{n}\right)\left\|x_{n}-T y_{n}\right\|^{2} \\
\leq & \left\|x_{n}-q\right\|^{2}-\alpha_{n} \beta_{n}\left(1-\beta_{n}\right)\left\|x_{n}-T x_{n}\right\|^{2} \\
\leq & \left\|x_{n}-q\right\|^{2} .
\end{aligned}
$$

Hence, we obtain that $\lim _{n \rightarrow \infty}\left\|x_{n}-z\right\|$ exists. This implies that $\left\{x_{n}\right\},\left\{y_{n}\right\}$, $\left\{T y_{n}\right\}$ are bounded. From 3.3, we know that

$$
\left\|x_{n+1}-q\right\|^{2} \leq\left\|x_{n}-q\right\|^{2}-\alpha_{n} \beta_{n}\left(1-\beta_{n}\right)\left\|x_{n}-T x_{n}\right\|^{2} .
$$

Since $0<a<\alpha_{n}<b<1$, we have that

$$
\left\|x_{n+1}-q\right\|^{2} \leq\left\|x_{n}-q\right\|^{2}-a \beta_{n}\left(1-\beta_{n}\right)\left\|x_{n}-T x_{n}\right\|^{2}
$$

we also know from $\lim \inf _{n \rightarrow \infty} \beta_{n}\left(1-\beta_{n}\right)>0$ that

$$
0 \leq a \beta_{n}\left(1-\beta_{n}\right)\left\|x_{n}-T x_{n}\right\|^{2} \leq\left\|x_{n}-q\right\|^{2}-\left\|x_{n+1}-q\right\|^{2} \rightarrow 0
$$

as $n \rightarrow \infty$. This means that

$$
\lim _{n \rightarrow \infty}\left\|x_{n}-T x_{n}\right\|=0 .
$$


Since $\left\{x_{n}\right\}$ is bounded, then, there exists a subsequence $\left\{x_{n_{i}}\right\}$ of $\left\{x_{n}\right\}$ such that $x_{n_{i}} \rightarrow x^{*} \in C$. Since $T$ is a generalized hybrid mapping, then

$$
\alpha\|T x-T y\|^{2}+(1-\alpha)\|x-T y\|^{2} \leq \beta\|T x-y\|^{2}+(1-\beta)\|x-y\|^{2}
$$

hence

$$
\begin{aligned}
0 \leq & \beta\|T x-y\|^{2}+(1-\beta)\|x-y\|^{2}-\alpha\|T x-T y\|^{2}-(1-\alpha)\|x-T y\|^{2} \\
\leq & \beta\left(\left\|T x_{n}\right\|^{2}-2\left\langle T x_{n}, x^{*}\right\rangle+\left\|x^{*}\right\|^{2}\right)+(1-\beta)\left(\left\|x_{n}\right\|^{2}-2\left\langle x_{n}, x^{*}\right\rangle+\left\|x^{*}\right\|^{2}\right) \\
& -\alpha\left(\left\|T x_{n}\right\|^{2}-2\left\langle T x_{n}, T x^{*}\right\rangle+\left\|T x^{*}\right\|^{2}\right)-(1-\alpha)\left(\left\|x_{n}\right\|^{2}-2\left\langle x_{n}, T x^{*}\right\rangle\right. \\
& \left.+\left\|T x^{*}\right\|^{2}\right) \\
= & \left\|x^{*}\right\|^{2}-\left\|T x^{*}\right\|^{2}+(\beta-\alpha)\left(\left\|T x_{n}\right\|^{2}-\left\|x_{n}\right\|^{2}\right) \\
& +2 \alpha\left\langle T x_{n}-x_{n}, T x^{*}\right\rangle-2 \beta\left\langle T x_{n}-x_{n}, x^{*}\right\rangle+2\left\langle x_{n}, T x^{*}-x^{*}\right\rangle \\
\leq & \left\|x^{*}\right\|^{2}-\left\|T x^{*}\right\|^{2}+(\beta-\alpha)\left(\left\|T x_{n}\right\|+\left\|x_{n}\right\|\right)\left(\left\|T x_{n}-x_{n}\right\|\right) \\
& +2 \alpha\left\langle T x_{n}-x_{n}, T x^{*}\right\rangle-2 \beta\left\langle T x_{n}-x_{n}, x^{*}\right\rangle+2\left\langle x_{n}, T x^{*}-x^{*}\right\rangle .
\end{aligned}
$$

Thus, we have that for all $i \in \mathbb{N}$,

$$
\begin{aligned}
0 \leq & \left\|x^{*}\right\|^{2}-\left\|T x^{*}\right\|^{2}+(\beta-\alpha)\left(\left\|T x_{n_{i}}\right\|+\left\|x_{n_{i}}\right\|\right)\left(\left\|T x_{n_{i}}-x_{n_{i}}\right\|\right) \\
& +2 \alpha\left\langle T x_{n_{i}}-x_{n_{i}}, T x^{*}\right\rangle-2 \beta\left\langle T x_{n_{i}}-x_{n_{i}}, x^{*}\right\rangle+2\left\langle x_{n_{i}}, T x^{*}-x^{*}\right\rangle .
\end{aligned}
$$

From 3.4, we have that

$$
\lim _{n \rightarrow \infty}\left\|x_{n_{i}}-T x_{n_{i}}\right\|=0
$$

and $x_{n_{i}} \rightarrow x^{*}$ as $i \rightarrow \infty$, the above inequality implies that

$$
\begin{aligned}
0 & \leq\left\|x^{*}\right\|^{2}-\left\|T x^{*}\right\|^{2}+2\left\langle x^{*}, T x^{*}-x^{*}\right\rangle \\
& =2\left\langle x^{*}, T x^{*}\right\rangle-\left\|x^{*}\right\|^{2}-\left\|T x^{*}\right\|^{2} \\
& =-\left\|x^{*}-T x^{*}\right\|^{2} .
\end{aligned}
$$

So, we have $T x^{*}=x^{*}$, i.e., $x^{*} \in F(T)$. Therefore we obtain that

$$
x^{*} \in F(T) \text {. }
$$

This implies that the condition (ii) of Lemma 2.3 holds for $S=F(T)$. We also know that $\lim _{n \rightarrow \infty}\left\|x_{n}-q\right\|$ exists for $q \in S=F(T)$. So, we have from Lemma 2.3 that there exists $x_{0} \in S$ such that $x_{n} \rightarrow x_{0}$ as $n \rightarrow \infty$. Moreover, since for any $q \in S=F(T)$,

$$
\left\|x_{n+1}-q\right\| \leq\left\|x_{n}-q\right\|, \quad \forall n \in \mathbb{N},
$$

by Lemma 2.2 there exists some $x \in S$ such that $P_{S}\left(x_{n}\right) \rightarrow x$. The property of metric projection implies that

$$
\left\langle x_{0}-P_{S}\left(x_{n}\right), x_{n}-P_{S}\left(x_{n}\right)\right\rangle \leq 0 .
$$

Therefore, we have

$$
\left\langle x_{0}-x, x_{0}-x\right\rangle=\left\|x_{0}-x\right\|^{2} \leq 0 .
$$


This means that $x_{0}=x$, i.e., $x_{n} \rightarrow x_{0}=\lim _{n \rightarrow \infty} P_{F(T)}\left(x_{n}\right)$.

Using Theorem 3.1 we prove the following weakly convergence theorem for nonspreading mappings in a Hilbert space.

Theorem 3.2. Let $C$ be a nonempty, closed convex subset of a real Hlibert space $H$. Let $T: C \rightarrow C$ be a nonspreading mapping with $F(T) \neq \emptyset$. Suppose that $\left\{x_{n}\right\}$ is a sequence generated by $x_{0}=x \in C, u \in C$ and

$$
\left\{\begin{array}{l}
y_{n}=\left(1-\beta_{n}\right) x_{n}+\beta_{n} T x_{n}, \\
x_{n+1}=\left(1-\alpha_{n}\right) x_{n}+\alpha_{n} T y_{n},
\end{array}\right.
$$

where $\left\{\alpha_{n}\right\}_{n=1}^{\infty}$ and $\left\{\beta_{n}\right\}_{n=1}^{\infty}$ are sequences of $[0,1]$ with $\liminf _{n \rightarrow \infty} \beta_{n}(1-$ $\left.\beta_{n}\right)>0$ and $0<a<\alpha_{n}<b<1$. Then $\left\{x_{n}\right\}$ converges weakly to $x_{0} \in F(T)$, where $x_{0}=\lim _{n \rightarrow \infty} P_{F(T)}\left(x_{n}\right)$.

Proof. Since $T: C \rightarrow C$ is an nonspreading mapping, then

$$
2\|S x-S y\|^{2} \leq\|S x-y\|^{2}+\|x-S y\|^{2} .
$$

The mapping $T$ is a $(2,1)$-generalized hybrid mapping. The result follows immediately from Theorem 3.1.

Remark. We also can prove the weakly convergence theorem for hybrid mappings as $\alpha=\frac{3}{2}, \beta=\frac{1}{2}$ and nonexpansive mappings as $\alpha=1, \beta=0$ in a Hilbert space.

\section{References}

[1] K. Aoyama, S. Iemoto, F. Kohsaka, and W. Takahashi, Fixed point and ergodic theorems for $\lambda$-hybrid mappings in Hilbert spaces, J. Nonlinear Convex Anal. 11 (2010), no. 2, $335-343$.

[2] E. Blum and W. Oettli, From optimization and variational inequalities to equilibrium problems, Math. Student 63 (1994), no. 1-4, 123-145.

[3] F. E. Browder, Convergence theorems for sequences of nonlinear operators in Banach spaces, Math. Z. 100 (1967), 201-225.

[4] P. L. Combettes and A. Hirstoaga, Equilibrium programming in Hilbert spaces, J. Nonlinear Convex Anal. 6 (2005), no. 1, 117-136.

[5] K. Goebel and W. A. Kirk, Topics in Metric Fixed Point Theory, Cambridge University Press, Cambridge, 1990.

[6] B. Halpern, Fixed points of nonexpanding maps, Bull. Amer. Math. Soc. 73 (1967), 957-961.

[7] M. Hojo, W. Takahashi, and I. Termwuttipong, Strong convergence theorems for 2generalized hybrid mappings in Hilbert spaces, Nonlinear Anal. 75 (2012), no. 4, 21662176.

[8] S. Iemoto and W. Takahashi, Approximating fixed points of nonexpansive mappings and nonspreading mappings in a Hilbert space, Nonlinear Anal. 71 (2009), no. 12, 2082-2089.

[9] S. Ishikawa, Fixed points by a new iteration, Proc. Amer. Math. Soc. 44 (1974), 147-150.

[10] S. Itoh and W. Takahashi, The common fixed point theory of single-valued mappings and multi-valued mappings, Pacific J. Math. 79 (1978), no. 2, 493-508. 
[11] F. Kohsaka and W. Takahashi, Fixed point theorems for a class of nonlinear mappings related to maximal monotone operators in Banach spaces, Arch. Math. 91 (2008), no. 2, 166-177.

[12] _ Existence and approximation of fixed points of firmly nonexpansive-type mappings in Banach spaces, SIAM J. Optim. 19 (2008), no. 2, 824-835.

[13] P. Kocourek, W. Takahashi, and J.-C. Yao, Fixed point theorems and weak convergence theorems for generalized hybrid mappings in Hilbert spaces, Taiwanese J. Math. 14 (2010), no. 6, 2497-2511.

[14] W. R. Mann, Mean value methods in iteration method, Proc. Amer. Math. Soc. 4 (1953), $506-510$.

[15] Z. Opial, Weak convergence of the sequence of successive approximations for nonexpansive mappings, Bull. Amer. Math. Soc. 73 (1967), 591-597.

[16] W. Takahashi, Nonlinear Functional Analysis, Yokohoma Publishers, Yokohama, 2000.

[17] _ Introduction to Nonlinear and Convex Analysis, Yokohama Publishers, Yokohama, 2005.

[18] W. Takahashi and M. Toyoda, Weak convergence theorems for nonexpansive mappings and monotone mappings, J. Optim. Theory Appl. 118 (2003), no. 2, 417-482.

[19] W. Takahashi and J.-C. Yao, Fixed point theorems and ergodic theorems for nonlinear mappings in Hilbert spaces, Taiwanese J. Math. 15 (2011), no. 2, 457-472.

FANGFANG YAN

Department of Mathematics

Tianjin Polytechnic University

Tianjin 300387, P. R. China

E-mail address: yanfangfang_2011@163.com

YONGFU SU

Department of Mathematics

Tianjin Polytechnic University

Tianjin 300387, P. R. China

E-mail address: suyongfu@tjpu.edu.cn

QINSHENG FENG

Department of Mathematics

Tianjin Polytechnic University

Tianjin 300387, P. R. China

E-mail address: fengqiansheng-2008@163.com 